Volume 28 (2020) 175-186

DOI: $10.24330 /$ ieja. 768246

\title{
ON THE RADICAL OF THE CENTER OF SMALL SYMMETRIC LOCAL ALGEBRAS
}

\author{
Pierre Landrock \\ Received: 31 October 2019; Revised: 8 January 2020; Accepted: 20 January 2020 \\ Communicated by A. Çiğdem Özcan
}

\begin{abstract}
This article is motivated by some results from Chlebowitz and Külshammer on how the structure of a symmetric local algebra is influenced by its center. They have shown that a symmetric local algebra is almost always commutative if its center is at most 5-dimensional. In this article we are interested in how the ideal property of the radical of the center of a symmetric local algebra is influenced by the dimension of the algebra itself.
\end{abstract}

Mathematics Subject Classification (2020): 16N40, 20C20

Keywords: Symmetric algebra, local algebra, radical of the center

\section{Introduction}

When studying the modular representation theory of a block $B$ of a group algebra $F G$ with $G$ being a finite group, it is of major interest to determine the numbers $k(B)$ of irreducible ordinary and $l(B)$ of irreducible Brauer characters. On the one hand there are numerous open conjectures on how these block invariants relate to each other and to the defect groups of $B$. On the other hand one can ask the following question: What can be said about the structure of $B$ itself if only $k(B)$, $l(B)$ and maybe the structure of the defect groups of $B$ are known? For instance, one can say that $k(B)$ is just the dimension of the center of $B$ and $l(B)$ is the number of isomorphism classes of simple $B$-modules in the modular case.

If one tries to study the representation theory of a block $B$ it is sometimes useful to replace $B$ by its basic algebra $A$. If $l(B)=1$ then $A$ is a symmetric local algebra. Hence, studying properties of symmetric local algebras adds to the knowledge about blocks of group algebras. Some more recent examples of this principle can be found in the literature, e.g. in [4], the Morita equivalence class of a whole family of 3-blocks with elementary abelian defect groups of order 9 was determined by investigating certain 9-dimensional symmetric local algebras. In [7] the authors were able to determine the isomorphism type of the center of a class of 2-blocks with elementary abelian defect groups of order 16. A big part of the 
argument was, again, based on investigating symmetric local algebras of a certain type.

In 1984, Külshammer proved that symmetric local algebras with a center being of dimension at most 4 have to be commutative (see [5]). Some years later, Chlebowitz and Külshammer could show that symmetric local algebras with 5-dimensional center have to be either of dimension 5 , and hence commutative, or of dimension 8 (see [1]). However, in order to obtain this result, a fair amount of computation was needed. These two articles suggest that there should be some more connections between a symmetric local algebra and its center, at least in low dimensions.

In this article we are interested in the question whether the radical of the center of a symmetric local algebra is an ideal of the whole algebra. Readers being familiar with the article [4] by Kessar will remember that showing this fact for a class of symmetric local algebras of dimension 9 was one of the crucial steps to get the computations going. In the present article we will, in fact, show that the radical of the center of a symmetric local algebra is an ideal of the whole algebra if the dimension of the algebra is at most 10 . Hence the corresponding fact in the article [4] would be an easy corollary from our main result.

The present article is organized as follows: In Section 2 we will gather some well known properties of symmetric local algebras. In the third section we will first prove a lemma which characterizes when the radical of the center of a symmetric local algebra is an ideal of the whole algebra. Moreover, this section will contain the main result of the present paper. In the final section we will apply a modification of the main result in characteristic 2 to the setting in [7]. This will allow us to considerably shorten the computations and give a more elegant proof of the main result of that article.

\section{Preliminaries}

Throughout this section $F$ will be an algebraically closed field (of arbitrary characteristic) and $A$ will be a finite dimensional $F$-algebra. We will denote the Jacobson radical of $A$ by $J:=J(A)$. Note that under our assumptions $A$ is local if and only if $\operatorname{dim}_{F}(A / J)=1$. Moreover, we will use the notation $Z(A)$ for the center of $A$ and $J(Z):=J(Z(A))$ for the Jacobson radical of the $F$-algebra $Z(A)$. For elements $x, y \in A$ we will denote their commutator by $[x, y]:=x y-y x$. For $F$-subspaces $U, V \subseteq A$ we set $[U, V]:=F\{[u, v] \mid u \in U, v \in V\}$. The commutator subspace of $A$ is given by $K(A):=[A, A]$.

By "ideal" we will always mean "two-sided ideal". 
If $A$ is symmetric, we fix a symmetrizing linear form $s: A \rightarrow F$ on $A$. For an $F$-subspace $U \subseteq A$ we denote its orthogonal space by $U^{\perp}:=\{x \in A \mid s(x U)=0\}$. The following lemma gathers some well-known facts about symmetric local algebras.

Lemma 2.1. Let $A$ be a symmetric local F-algebra. Then the following facts hold.

(i) For an F-subspace $U \subseteq A$ we have $\operatorname{dim}_{F} U+\operatorname{dim}_{F} U^{\perp}=\operatorname{dim}_{F} A$. Moreover, the identity $\left(U^{\perp}\right)^{\perp}=U$ holds.

(ii) For F-subspaces $U, V \subseteq A$ we have $(U \cap V)^{\perp}=U^{\perp}+V^{\perp}$.

(iii) We have $J^{\perp}=\operatorname{soc}(A)$ and $K(A)^{\perp}=Z(A)$.

(iv) $Z(A)$ is a local $F$-algebra and $J(Z)=Z(A) \cap J$.

(v) We have $\operatorname{dim}_{F}(\operatorname{soc}(A))=1$.

(vi) We have $K(A) \cap \operatorname{soc}(A)=0$.

(vii) If $I$ is an ideal of $A$ then $I^{\perp}$ also is an ideal of $A$ and $I \cdot I^{\perp}=I^{\perp} \cdot I=0$.

(viii) If $n \in \mathbb{N}_{0}$ is minimal with $J^{n+1}=0$ then $J^{n}=\operatorname{soc}(A)$.

Proof. The first two items are well-known properties of non-degenerate symmetric bilinear forms. Note that $\lambda: A \times A \rightarrow F,(x, y) \mapsto s(x y)$, defines such a form on $A$. The definition of $\operatorname{soc}(A)$ implies $\operatorname{soc}(A) \subseteq J^{\perp}$. Moreover, from the first part of (i) we deduce $\operatorname{dim}_{F} J^{\perp}=1$. Since $\operatorname{soc}(A) \neq 0$, one immediately obtains the first part of (iii), as well as (v). The second part of (iii) is proved in [5, Lemma C]. The proofs for (iv), (vi), and (viii) of can be found in [4]. Thus, only (vii) needs to be proved.

Let $I$ be an ideal of $A$, and let $x \in I^{\perp}$ and $a \in A$ be arbitrary. Then

$$
s((x a) I)=s(x(a I)) \subseteq s(x I)=0,
$$

by the ideal property of $I$ and the definition of $I^{\perp}$. This shows $x a \in I^{\perp}$. On the other hand,

$$
s((a x) I))=s(a(x I))=s((x I) a)=s(x(I a)) \subseteq s(x I)=0,
$$

again by the ideal property of $I$ and the definition of $I^{\perp}$. This shows $a x \in I^{\perp}$. Thus, we have proved that $I^{\perp}$ is an ideal of $A$. We deduce further

$$
0=s\left(I \cdot I^{\perp}\right)=s\left(\left(I \cdot I^{\perp}\right) \cdot A\right) .
$$

From this, we conclude $I \cdot I^{\perp} \subseteq A^{\perp}=0$. Similarly, we obtain $I^{\perp} \cdot I=0$. This finishes the proof.

We need another lemma which gives a characterization of when a factor algebra of a symmetric algebra is again symmetric. 
Lemma 2.2. Let $A$ be a symmetric F-algebra and $I$ be an ideal of $A$. Then the $F$-algebra $A / I$ is again symmetric if and only if $I=(A z)^{\perp}$ for some element $z \in Z(A)$.

Proof. See [2, Theorem 2.6].

From Lemma 2.2 we immediately get the following corollary.

Corollary 2.3. Let $A$ be a symmetric local $F$-algebra and $0 \neq z \in Z(A)$. Then $A /(A z)^{\perp}$ also is a symmetric local $F$-algebra.

Proof. By Lemma 2.2 we just need to show that $A^{\prime}:=A /(A z)^{\perp}$ is local. But since $A$ is local we know that $\operatorname{dim}_{F}(A / J)=1$. Hence it is enough to show that $(A z)^{\perp} \subseteq J$ holds. For, from $(A z)^{\perp} \subseteq J$ we obtain $J\left(A^{\prime}\right)=J /(A z)^{\perp}$ and thus $A^{\prime} / J\left(A^{\prime}\right) \cong A / J$. Hence $\operatorname{dim}_{F}\left(A^{\prime} / J\left(A^{\prime}\right)\right)=\operatorname{dim}_{F}(A / J)=1$ and $A^{\prime}=A /(A z)^{\perp}$ is local.

Since $0 \neq z \in A z$, we have $0 \neq A z$, which implies $(A z)^{\perp} \neq A$. Since $A$ is local and $(A z)^{\perp}$ is a proper ideal of $A$, we immediately get $(A z)^{\perp} \subseteq J$.

Next we will recap a result which gives a nice way of computing a generating set of a Loewy-layer of a given algebra if a generating set of the previous Loewy-layer is known.

Lemma 2.4. Let $A$ be an F-algebra, let $I$ be an ideal of $A$ and let $n \in \mathbb{N}$. Suppose that there is some $d \in \mathbb{N}$ such that

$$
I^{n}=F\left\{x_{i 1} \ldots x_{i n} \mid i=1, \ldots, d\right\}+I^{n+1}
$$

with elements $x_{i k} \in I$. Then we have

$$
I^{n+1}=F\left\{x_{j 1} x_{i 1} \ldots x_{i n} \mid i, j=1, \ldots, d\right\}+I^{n+2} .
$$

Proof. This is a slightly simplified version of [5, Lemma E].

As a last fact in this section we recap one of the results mentioned in the introduction of this article.

Lemma 2.5. Let $A$ be a symmetric local $F$-algebra. If $\operatorname{dim}_{F} Z(A) \leq 4$ then $A$ is commutative.

Proof. See [5, Theorem B]. 


\section{The main result}

In this section $F$ will again be an algebraically closed field and $A$ will be a finite dimensional $F$-algebra. First we will point out a characterization of when the radical (resp. the socle) of the center of a symmetric local algebra $A$ is an ideal of $A$. Although not hard to prove, this result seems to be new so we will give a proof here.

Lemma 3.1. Let $A$ be a symmetric local F-algebra.

(i) $J(Z)$ is an ideal of $A$ if and only if $K(A) \cdot J(Z)=0$.

(ii) $\operatorname{soc}(Z(A))$ is an ideal of $A$ if and only if $K(A) \cdot \operatorname{soc}(Z(A))=0$.

Proof. (i) Let $J(Z)$ be an ideal of $A$. Then $J(Z)=Z(A) \cap J$, by (iv) of Lemma 2.1. Switching to orthogonal spaces and using (ii), (iii), (iv), (vi) and (vii) of Lemma 2.1 , we obtain that

$$
J(Z)^{\perp}=(Z(A) \cap J)^{\perp}=Z(A)^{\perp}+J^{\perp}=K(A) \oplus \operatorname{soc}(A)
$$

is an ideal of $A$ and $K(A) \cdot J(Z) \subseteq J(Z)^{\perp} \cdot J(Z)=0$.

Next, we assume that $K(A) \cdot J(Z)=0$ holds. Let $a, b \in A$ and $z \in J(Z)$ be arbitrary. Then $0=(a b-b a) z=(a z) b-b(a z)$. Since $b \in A$ was arbitrary and $z \in J(Z) \subseteq J$, we conclude $a z \in Z(A) \cap J=J(Z)$. But since $a \in A$ and $z \in J(Z)$ were arbitrary too, this implies $J(Z) \cdot A=A \cdot J(Z) \subseteq J(Z)$. This shows the claim.

(ii) The claim is trivial if $\operatorname{dim}_{F} Z(A)=1$. For, by Lemma 2.5 this identity implies $A=Z(A)=\operatorname{soc}(Z(A))$ and therefore $K(A)=0$. Thus we may assume $\operatorname{dim}_{F} Z(A)>1$. Hence, by (iv) of Lemma 2.1, we conclude $\operatorname{soc}(Z(A)) \subseteq J(Z)$.

First, let us assume that $\operatorname{soc}(Z(A))$ is an ideal of $A$. We obtain

$$
K(A) \oplus \operatorname{soc}(A)=J(Z)^{\perp} \subseteq \operatorname{soc}(Z(A))^{\perp}
$$

and this implies $K(A) \cdot \operatorname{soc}(Z(A))=0$ with the same argument as in the proof of the first item.

Now we assume that $K(A) \cdot \operatorname{soc}(Z(A))=0$ holds. Therefore, for arbitrary $a, b \in A$ and $z \in \operatorname{soc}(Z(A)) \subseteq J(Z)$ we get $0=(a b-b a) z=(a z) b-b(a z)$. Hence we must have $a z \in Z(A) \cap J=J(Z)$ since $b \in A$ was arbitrary and $z \in J(Z) \subseteq J$. Using that $a \in A$ and $z \in \operatorname{soc}(Z(A))$ were arbitrary too, we obtain $A \cdot \operatorname{soc}(Z(A)) \subseteq J(Z)$. Furthermore, we have $(A \cdot \operatorname{soc}(Z(A))) \cdot J(Z)=0$ since we already have $\operatorname{soc}(Z(A))$. $J(Z)=0$. Hence $A \cdot \operatorname{soc}(Z(A)) \subseteq J(Z)$ is annihilated by $J(Z)$ and must therefore be contained in $\operatorname{soc}(Z(A))$. Thus $\operatorname{soc}(Z(A)) \cdot A=A \cdot \operatorname{soc}(Z(A)) \subseteq \operatorname{soc}(Z(A))$ which shows the claim. 
Next, we will use Lemma 3.1 to show the main result of this article.

Theorem 3.2. Let $A$ be a symmetric local $F$-algebra. If $\operatorname{dim}_{F} A \leq 10$ then $J(Z)$ is an ideal of $A$.

Proof. Assume $\operatorname{dim}_{F} A \leq 10$ and that $J(Z)$ is not an ideal of $A$. Hence, by Lemma 3.1 there is some $0 \neq z \in J(Z)$ with $z K(A) \neq 0$. In particular, we have $(A z) \cdot K(A) \neq 0$ so that $K(A) \nsubseteq(A z)^{\perp}$. In the following, we will consider the $F$ algebra $A^{\prime}:=A /(A z)^{\perp}$ and try to constrain its dimension from below. By Corollary 2.3 we know that $A^{\prime}$ is also symmetric and local and by (i) of Lemma 2.1 we have

$$
\operatorname{dim}_{F}(A z)=\operatorname{dim}_{F} A-\operatorname{dim}_{F}(A z)^{\perp}=\operatorname{dim}_{F} A^{\prime} .
$$

Since $K(A) \nsubseteq(A z)^{\perp}$, we have $K\left(A^{\prime}\right) \neq 0$. In particular, $A^{\prime}$ is not commutative. By Lemma 2.5 we immediately get

$$
\operatorname{dim}_{F} Z\left(A^{\prime}\right) \geq 5
$$

We will now show that $\operatorname{dim}_{F} A^{\prime} \geq \operatorname{dim}_{F} Z\left(A^{\prime}\right)+3$ holds. Since $A^{\prime}$ is not commutative, we already know $\operatorname{dim}_{F} A^{\prime} \geq \operatorname{dim}_{F} Z\left(A^{\prime}\right)+1$.

Assume that $\operatorname{dim}_{F} A^{\prime}=\operatorname{dim}_{F} Z\left(A^{\prime}\right)+1$. Then we find some $x^{\prime} \in A^{\prime}$ such that $A^{\prime}=F x^{\prime} \oplus Z\left(A^{\prime}\right)$. But this implies

$$
K\left(A^{\prime}\right)=\left[F x^{\prime}+Z\left(A^{\prime}\right), F x^{\prime}+Z\left(A^{\prime}\right)\right]=0
$$

and hence $A^{\prime}$ must be commutative, a contradiction.

Next, assume that $\operatorname{dim}_{F} A^{\prime}=\operatorname{dim}_{F} Z\left(A^{\prime}\right)+2$. Similarly to the previous case, we find $x^{\prime}, y^{\prime} \in A^{\prime}$ such that $A^{\prime}=F x^{\prime} \oplus F y^{\prime} \oplus Z\left(A^{\prime}\right)$. Hence

$$
K\left(A^{\prime}\right)=\left[F x^{\prime}+F y^{\prime}+Z\left(A^{\prime}\right), F x^{\prime}+F y^{\prime}+Z\left(A^{\prime}\right)\right] \subseteq F\left[x^{\prime}, y^{\prime}\right] .
$$

But then, using (i) and (iii) of Lemma 2.1, we obtain

$$
\begin{aligned}
\operatorname{dim}_{F} Z\left(A^{\prime}\right) & =\operatorname{dim}_{F} A^{\prime}-2 \\
& =\operatorname{dim}_{F} Z\left(A^{\prime}\right)+\operatorname{dim}_{F} K\left(A^{\prime}\right)-2 \\
& \leq \operatorname{dim}_{F} Z\left(A^{\prime}\right)+1-2 \\
& =\operatorname{dim}_{F} Z\left(A^{\prime}\right)-1,
\end{aligned}
$$

a contradiction. This shows that $\operatorname{dim}_{F} A^{\prime} \geq \operatorname{dim}_{F} Z\left(A^{\prime}\right)+3$ holds. Combining this result with (1) and (2), we obtain $\operatorname{dim}_{F}(A z) \geq 5+3=8$. Since $A$ is a local $F$-algebra, we have $A=F 1 \oplus J$. Therefore, using $z \in J(Z)$ we obtain $A z \subseteq F z+J \cdot J(Z) \subseteq F z+J^{2} \subseteq J$. Since $F 1 \oplus A z \subseteq A$ and $\operatorname{dim}_{F}(A z) \geq 8$, we conclude $\operatorname{dim}_{F} A \in\{9,10\}$. We distinguish these two cases. 
First, let $\operatorname{dim}_{F} A=9$. Then we must have $A=F 1 \oplus A z \subseteq F 1+F z+J^{2}$ and $J / J^{2}$ is spanned by $\left\{z+J^{2}\right\}$. Using Lemma 2.4 , this implies that $A$ is generated, as an $F$-algebra, by $\{1, z\}$. Since $1, z \in Z(A)$, this means that $A$ must be commutative. Hence $A=Z(A)$ and $J(Z)=J$ is an ideal of $A$, a contradiction to our assumption.

Finally, let $\operatorname{dim}_{F} A=10$. Similarly to the previous case, we deduce that $A=$ $F 1+F x+F z+J^{2}$ for some $x \in J$. Thus, $J / J^{2}$ is spanned by a subset of $\left\{x+J^{2}, z+J^{2}\right\}$ and $A$ is generated, as an $F$-algebra, by a subset of $\{1, x, z\}$. Since $1, z \in Z(A)$, all the generators of $A$ commute and thus $A$ is commutative. We get a contradiction with the same argument as before. This finishes the proof.

Remark 3.3. Comparing the statement of Theorem 3.2 with that of Lemma 2.5 or that of the main theorem of [1], the reader will notice some analogy. While in Lemma 2.5 or [1] a bound on the dimension of the center of an algebra is used to obtain structural results on the whole algebra, Theorem 3.2 uses a bound on the dimension of the algebra itself to specify the relationship between an algebra and its center.

\section{An application}

In this section we are revisiting the article [7], especially its fourth section. The article mentioned is concerned with determining the isomorphism type of the center of a non-nilpotent 2-block $B$ with elementary abelian defect group of order 16 and one isomorphism type of simple modules. By computing all the possible generalized decomposition matrices in the first part of that article, the authors show that the center of $B$ must have one of two possible isomorphism types (see [7, Proposition 2.1]). In the following two sections they rule out one of those two possibilities leaving one unique isomorphism type for the center of $B$. That part of the paper is heavily computational and not too illuminating.

In fact, Charles Eaton has classified the 2-blocks with elementary abelian defect group of order 16, up to Morita equivalence (see [3]). The resulting list contains just one non-nilpotent block with exactly one isomorphism type of simple modules. His approach, however, relies on the classification of finite simple groups. The approach taken in the article [7] and the thesis [6] can be seen as a classification-free attempt to determine the structure of the unique non-nilpotent 2-block with elementary abelian defect group of order 16 and one isomorphism type of simple modules.

We will, in this section, prove a stronger result of our Theorem 3.2 which, on the other hand, needs some more restrictive assumptions. Using this result, we can give another more elegant proof that one of the two isomorphism types of the 
center given in [7, Proposition 2.1] cannot occur. For this proof, we will only need [7, Lemma 4.1], which we will quote below, and [7, Lemma 4.2 (i),(iv)] from the article mentioned. Moreover, the reader should note that the proof of $[7$, Lemma 4.2 (iv)] is independent of [7, Lemma 4.2 (ii),(iii)].

From now on, $F$ will denote an algebraically closed field of characteristic 2 and $B$ will be a non-nilpotent block with elementary abelian defect group of order 16 and one isomorphism type of simple modules. Moreover, $A$ will denote a basic algebra of $B$. We will, again, use $J:=J(A)$ and $J(Z):=J(Z(A))$. First we obtain

Lemma 4.1. Let $F, B$ and $A$ be as above. Then

(i) $\operatorname{dim}_{F} A=16$ and $\operatorname{dim}_{F} Z(A)=\operatorname{dim}_{F} K(A)=8$.

(ii) $A$ is a symmetric local $F$-algebra.

(iii) $Z(A) \cong Z(B)$.

(iv) For every $w \in J$ we have $w^{2} \in K(A)$.

Proof. This is part of [7, Lemma 4.1].

As in the article [7], we will now assume that $Z(A)=F\left\{W_{0}:=1, W_{1}, \ldots, W_{7}\right\}$ where the structure constants are given by the following table.

\begin{tabular}{|c|cccccccc|}
\hline$\cdot$ & 1 & $W_{1}$ & $W_{2}$ & $W_{3}$ & $W_{4}$ & $W_{5}$ & $W_{6}$ & $W_{7}$ \\
\hline 1 & 1 & $W_{1}$ & $W_{2}$ & $W_{3}$ & $W_{4}$ & $W_{5}$ & $W_{6}$ & $W_{7}$ \\
$W_{1}$ & $W_{1}$ &. & $W_{5}$ & $W_{6}$ & $W_{7}$ &. &. &. \\
$W_{2}$ & $W_{2}$ & $W_{5}$ &. &. &. &. &. &. \\
$W_{3}$ & $W_{3}$ & $W_{6}$ &. &. &. &. &. &. \\
$W_{4}$ & $W_{4}$ & $W_{7}$ &. &. &. &. &. &. \\
$W_{5}$ & $W_{5}$ &. &. &. &. &. &. &. \\
$W_{6}$ & $W_{6}$ &. &. &. &. &. &. &. \\
$W_{7}$ & $W_{7}$ &. &. &. &. &. &. &. \\
\hline
\end{tabular}

We immediately obtain $J(Z)=F\left\{W_{1}, \ldots, W_{7}\right\}$. this, in turn, implies the identity $\operatorname{soc}(Z(A))=J(Z)^{2}=F\left\{W_{5}, W_{6}, W_{7}\right\}$. In particular, we have $\operatorname{dim}_{F} J(Z)=7$ and $\operatorname{dim}_{F} J(Z)^{2}=3$. In addition, from $W_{i}^{2}=0$ for $i=1, \ldots, 7$ and $\operatorname{char}(F)=2$ we conclude $z^{2}=0$ for any $z \in J(Z)$. The aim of this section is to prove that the assumption of this isomorphism type for $Z(A)$ leads to a contradiction. In order to do that, we first prove an analogue to Theorem 3.2 for the case we are considering in this section.

Theorem 4.2. Let $F, A$ and the isomorphism type of $Z(A)$ be as above. Then $J(Z)$ is an ideal of $A$. 
Proof. Assume that $J(Z)$ is not an ideal of $A$. Then we find some $0 \neq z \in J(Z)$ such that $z K(A) \neq 0$ by Lemma 3.1. Following the same arguments as in the proof of Theorem 3.2, one can show that $\operatorname{dim}_{F}(A z) \geq 8$. Since $z \in J(Z)$, we have $z^{2}=0$ and this implies $(A z)^{2}=A z^{2}=0$. Hence, we obtain $A z \subseteq(A z)^{\perp}$. Together with $16=\operatorname{dim}_{F} A=\operatorname{dim}_{F}(A z)+\operatorname{dim}_{F}(A z)^{\perp}$ and $\operatorname{dim}_{F}(A z) \geq 8$, this implies $\operatorname{dim}_{F}(A z)=8$ and $A z=(A z)^{\perp}$.

We continue by defining $A^{\prime}:=A /(A z)^{\perp}=A /(A z)$ as in the proof of Theorem 3.2. Following the lines of that proof again, we see that $\operatorname{dim}_{F} Z\left(A^{\prime}\right) \geq 5$, as well as $\operatorname{dim}_{F} A^{\prime} \geq \operatorname{dim}_{F} Z\left(A^{\prime}\right)+3$ and $\operatorname{dim}_{F} A^{\prime}=\operatorname{dim}_{F}(A z)=8$ hold. Thus, we get $\operatorname{dim}_{F} Z\left(A^{\prime}\right)=5$. Hence, $\operatorname{dim}_{F} K\left(A^{\prime}\right)=8-5=3$ and we obtain

$$
\begin{aligned}
\operatorname{dim}_{F}(K(A)+A z) & =\operatorname{dim}_{F}((K(A)+A z) / A z)+\operatorname{dim}_{F}(A z) \\
& =\operatorname{dim}_{F} K\left(A^{\prime}\right)+\operatorname{dim}_{F}(A z) \\
& =11 .
\end{aligned}
$$

Switching to orthogonal spaces and using $A z=(A z)^{\perp}$, we obtain the identity $\operatorname{dim}_{F}(Z(A) \cap(A z))=16-11=5$. Keeping in mind that $z^{2}=0$, we easily verify that the map $\varphi: Z(A)+A z \rightarrow z Z(A), a \mapsto z a$, is a well defined epimorphism of $F$-vector spaces with kernel $A z$. For, we have

$$
\text { ker } \varphi \subseteq\{a \in A \mid z a=0\}=\{a \in A \mid a(A z)=0\} \subseteq(A z)^{\perp}=A z \subseteq \operatorname{ker} \varphi
$$

Hence, we obtain an isomorphism

$$
\varphi^{\prime}:(Z(A)+A z) /(A z) \rightarrow z Z(A), a+(A z) \mapsto z a,
$$

of $F$-vector spaces. This implies

$$
\begin{aligned}
\operatorname{dim}_{F}(z Z(A)) & =\operatorname{dim}_{F}((Z(A)+A z) /(A z)) \\
& =\operatorname{dim}_{F}(Z(A) /(Z(A) \cap(A z))) \\
& =8-5 \\
& =3 .
\end{aligned}
$$

On the other hand, we have $Z(A)=F 1 \oplus J(Z)$. Hence, $z Z(A)=F z \oplus z J(Z)$. For, $z \in z J(Z)$ would imply $z(1-w)=0$ for some $w \in J(Z)$. Since $1-w$ is invertible, we infer $z=0$, contradicting to our choice of $z$. Thus, we have obtained $\operatorname{dim}_{F}(z J(Z))=\operatorname{dim}_{F}(z Z(A))-1=2$. Let us now write $z=\sum_{i=1}^{7} \lambda_{i} W_{i}$ with $\lambda_{i} \in F$ for $i=1, \ldots, 7$. Using the table with the structure constants of $Z(A)$, we 
easily see

$$
\operatorname{dim}_{F}(z J(Z))= \begin{cases}3: & \lambda_{1} \neq 0 \\ 1: & \lambda_{1}=0 \text { and }\left(\lambda_{2}, \lambda_{3}, \lambda_{4}\right) \neq(0,0,0) \\ 0: & \text { else. }\end{cases}
$$

In particular, we always have $\operatorname{dim}_{F}(z J(Z)) \neq 2$. But this contradicts our computation above where we have shown that $\operatorname{dim}_{F}(z J(Z))=2$ must hold. This shows that our assumption from the beginning must be wrong and finishes the proof.

Next, we will prove a lemma which will enable us to show that the ideal $J(Z)$ is annihilated by $J^{3}$. This will be the key ingredient for showing that the assumed isomorphism type of $Z(A)$ leads to a contradiction. In the following, we will make extensive use of Lemma 2.4 for computing generating sets of Loewy-layers without explicitly quoting this lemma every single time.

Lemma 4.3. Let $F, A$ and the isomorphism type of $Z(A)$ be as above. Moreover, let $z \in J(Z)$. Then $J^{3} \subseteq(A z)^{\perp}$.

Proof. Since the statement is obvious for $z=0$, we may assume $z \neq 0$ in the following. Let us assume that $J^{3} \nsubseteq(A z)^{\perp}$. Again, we define $A^{\prime}:=A /(A z)^{\perp}$ and use the notation $J^{\prime}:=J\left(A^{\prime}\right)$. Then we have $\left(J^{\prime}\right)^{3}=\left(J^{3}+(A z)^{\perp}\right) /(A z)^{\perp} \neq 0$. In particular, we have $\operatorname{dim}_{F}\left(J^{\prime}\right)^{3} \geq 1$. By Theorem 4.2, we have $A z \subseteq Z(A)$ and thus $K(A) \subseteq(A z)^{\perp}$. Therefore, $A^{\prime}$ must be a commutative $F$-algebra and, by (iv) of Lemma 4.1, we have $\left(w^{\prime}\right)^{2}=0$ for any $w^{\prime} \in J^{\prime}$.

Now assume that $\operatorname{dim}_{F}\left(\left(J^{\prime}\right)^{2} /\left(J^{\prime}\right)^{3}\right)=1$. Then $\left(J^{\prime}\right)^{2}=F x^{\prime} y^{\prime}+\left(J^{\prime}\right)^{3}$ for some $x^{\prime}, y^{\prime} \in J^{\prime}$. But from this and the note above, we get $\left(J^{\prime}\right)^{3}=F\left(x^{\prime}\right)^{2} y^{\prime}+\left(J^{\prime}\right)^{4}=\left(J^{\prime}\right)^{4}$ which, by Nakayama's lemma, implies $\left(J^{\prime}\right)^{3}=0$, a contradiction.

Hence, we may assume $\operatorname{dim}_{F}\left(\left(J^{\prime}\right)^{2} /\left(J^{\prime}\right)^{3}\right) \geq 2$. Using Lemma 2.4, we conclude that also $\operatorname{dim}_{F}\left(J^{\prime} /\left(J^{\prime}\right)^{2}\right) \geq 2$ must hold. Assuming $\operatorname{dim}_{F}\left(J^{\prime} /\left(J^{\prime}\right)^{2}\right)=2$, we could write $J^{\prime}=F x^{\prime}+F y^{\prime}+\left(J^{\prime}\right)^{2}$ with certain $x^{\prime}, y^{\prime} \in J^{\prime}$. However, using the notes from the start of the proof, this would imply $\left(J^{\prime}\right)^{2}=F x^{\prime} y^{\prime}+\left(J^{\prime}\right)^{3}$, contradicting $\operatorname{dim}_{F}\left(J^{\prime}\right)^{2} /\left(J^{\prime}\right)^{3} \geq 2$. Hence, we must even have $\operatorname{dim}_{F}\left(J^{\prime} /\left(J^{\prime}\right)^{2}\right) \geq 3$. Since $A$ is symmetric and local, we conclude from Lemma 2.2 and $z \neq 0$ that $A^{\prime}$ is also symmetric and local. Using (v) and (viii) of Lemma 2.1 and the inequality $\operatorname{dim}_{F}\left(\left(J^{\prime}\right)^{2} /\left(J^{\prime}\right)^{3}\right) \geq 2$, we obtain $\operatorname{dim}_{F}\left(\left(J^{\prime}\right)^{3} /\left(J^{\prime}\right)^{4}\right) \geq 1$. In particular, we have $\operatorname{dim}_{F} A^{\prime} \geq 1+3+2+1=7$. Since, on the other hand, the inequality $\operatorname{dim}_{F} A^{\prime}=\operatorname{dim}_{F}(A z) \leq \operatorname{dim}_{F} J(Z)=7$ does also hold, we must have $\operatorname{dim}_{F} A^{\prime}=7$ and $J(Z)=A z$. But this implies $J(Z)^{2}=(A z)^{2}=A z^{2}=0$ contradicting 
$\operatorname{dim}_{F} J(Z)^{2}=3$. This shows that our assumption is wrong and we indeed have $J^{3} \subseteq(A z)^{\perp}$.

We immediately obtain the following.

Corollary 4.4. Let $F, A$ and the isomorphism type of $Z(A)$ be as above. Then $J^{3} \subseteq J(Z)^{\perp}$. In particular, $J^{3} \cdot J(Z)=0$.

Proof. By Theorem 4.2, we have $J(Z)=\sum_{z \in J(Z)} A z$. Switching to orthogonal spaces, we get $J(Z)^{\perp}=\bigcap_{z \in J(Z)}(A z)^{\perp}$. Using Lemma 4.3 , we immediately get $J^{3} \subseteq \bigcap_{z \in J(Z)}(A z)^{\perp}=J(Z)^{\perp}$. The second part of the statement follows from Theorem 4.2 and (vii) of Lemma 2.1.

At this point, we quote another result from the article [7] which we will need.

Lemma 4.5. Let $F, A$ and the isomorphism type of $Z(A)$ be as above. Then $\operatorname{dim}_{F}\left(\left(J(Z)+J^{2}\right) / J^{2}\right) \leq 2$.

Proof. This is [7, Lemma 4.2 (iv)].

Now we have everything we need to prove the desired theorem.

Theorem 4.6. Let $F$ and $A$ be as in the beginning of this section. Then $Z(A)$ cannot have the isomorphism type given after Lemma 4.1.

Proof. Assume that $Z(A)$ has the isomorphism type given after Lemma 4.1. By Corollary 4.4, we have $J^{3} \cdot J(Z)=0$. In particular, $J^{2} \cdot J(Z) \subseteq J^{\perp}=\operatorname{soc}(A)$. By Lemma 4.5, we have $\operatorname{dim}_{F}\left(\left(J(Z)+J^{2}\right) / J^{2}\right) \leq 2$.

First, let us assume $\operatorname{dim}_{F}\left(\left(J(Z)+J^{2}\right) / J^{2}\right)=0$. Then $J(Z) \subseteq J^{2}$. However, this implies $J(Z)^{2} \subseteq J^{2} \cdot J(Z) \subseteq \operatorname{soc}(A)$ contradicting $\operatorname{dim}_{F} J(Z)^{2}=3$.

Next, assume that $\operatorname{dim}_{F}\left(\left(J(Z)+J^{2}\right) / J^{2}\right)=1$. Then there is some $z \in J(Z)$ such that $J(Z)=F z+\left(J(Z) \cap J^{2}\right)$. Using $z^{2}=0$, this implies

$$
J(Z)^{2} \subseteq F z^{2}+J^{2} \cdot J(Z) \subseteq J^{2} \cdot J(Z) \subseteq \operatorname{soc}(A),
$$

a contradiction as before.

Finally, let $\operatorname{dim}_{F}\left(\left(J(Z)+J^{2}\right) / J^{2}\right)=2$. Then we find $z_{1}, z_{2} \in J(Z)$ with $J(Z)=F z_{1}+F z_{2}+\left(J(Z) \cap J^{2}\right)$. Keeping in mind that $z_{1}^{2}=z_{2}^{2}=0$ and $z_{1} z_{2}=z_{2} z_{1}$, we obtain $J(Z)^{2} \subseteq F z_{1} z_{2}+\left(J^{2} \cdot J(Z)\right) \subseteq F z_{1} z_{2}+\operatorname{soc}(A)$. Since the right hand side is at most 2-dimensional over $F$, this is again a contradiction. Thus our assumption from the beginning of the proof cannot hold and we have shown the claim. 
Acknowledgement. The author is grateful to B. Külshammer for supervising his $\mathrm{PhD}$ thesis and encouraging him to publish this note which basically is an extraction from the mentioned $\mathrm{PhD}$ thesis. The author would also like to thank the referee for a number of useful comments for improving the quality of this note.

\section{References}

[1] M. Chlebowitz and B. Külshammer, Symmetric local algebras with 5dimensional center, Trans. Amer. Math. Soc., 329(2) (1992), 715-731.

[2] T. C. Craven and T. L. Smith, Symmetric algebras over rings and fields, Bull. Aust. Math. Soc., 89(3) (2014), 466-472.

[3] C. W. Eaton, Morita equivalence classes of blocks with elementary abelian defect groups of order 16, preprint, arXiv:1612.03485v4, (2019).

[4] R. Kessar, On blocks stably equivalent to a quantum complete intersection of dimension 9 in characteristic 3 and a case of the Abelian Defect Group Conjecture, J. Lond. Math. Soc., 85(2) (2012), 491-510.

[5] B. Külshammer, Symmetric local algebras and small blocks of finite groups, J. Algebra, 88 (1984), 190-195.

[6] P. Landrock, Eine Klasse von Blöcken mit Einer Elementarabelschen Defektgruppe der Ordnung 16, Ph.D. Thesis, Jena 2018.

[7] P. Landrock and B. Sambale, On centers of blocks with one simple module, J. Algebra, 472 (2017), 339-368.

Pierre Landrock

formerly:

Institute for Mathematics

University of Jena

07737 Jena, Germany

email: pierre.landrock@web.de 